\title{
Magnetic resonance direct thrombus imaging was accurate in suspected deep venous thrombosis
}

\author{
Fraser DG, Moody AR, Morgan PS, et al. Diagnosis of lower-limb deep venous thrombosis: a prospective blinded study of \\ magnetic resonance direct thrombus imaging. Ann Intern Med 2002 Jan 15;136:89-98.
}

\section{QUESTION: In patients with suspected deep venous thrombosis (DVT), is magnetic resonance direct thrombus imaging (MRDTI) an accurate diagnostic test?}

Design

Blinded comparison of MRDTI with venography.

\section{Setting}

A university based hospital in Nottingham, UK.

Source of funding: British Heart Foundation.

For correspondence: Dr A Moody, University Hospital, Nottingham, UK.

Alan.Moody@ nottingham.ac.uk.

\section{Patients}

101 eligible patients (age range 20 to 95 y) from a group of 338 consecutive patients with suspected DVT who had routine venography. Patients with a positive result on venography and a subset of $25 \%$ of patients with a negative result, selected according to a predetermined sequence, were included. Exclusion criteria were pregnancy, known contrast allergy, renal failure, no leg symptoms, failed or inconclusive venography or MRDTI, contraindications to magnetic resonance imaging, or claustrophobia.

Diagnostic characteristics for magnetic resonance direct thrombus imaging in suspected deep venous thrombosis (DVT)

\begin{tabular}{|c|c|c|c|c|}
\hline Disease & $\begin{array}{l}\text { Sensitivity } \\
(95 \% \mathrm{CI})\end{array}$ & Specificity (CI) & +LR & -LR \\
\hline All DVT & $96 \%$ (89 to 99$)$ & $90 \%$ (79 to 96$)$ & 9.24 & 0.04 \\
\hline Isolated calf DVT† & $92 \%(66$ to 100$)$ & $90 \%$ (77 to 97$)$ & 8.8 & 0.09 \\
\hline Proximal DVT & $98 \%$ (87 to 100$)$ & $100 \%(94$ to 100$)$ & $\infty$ & 0.02 \\
\hline
\end{tabular}

*Diagnostic terms defined in glossary; sensitivity, specificity, and Cls for isolated calf and proximal DVT calculated from data in article; LRs for all results calculated from data in article.

†Based on 60 patients with isolated calf DVT or no DVT.
Description of test and diagnostic standard MRDTI was done within 48 hours of venography. The scans were interpreted by an experienced radiologist and a non-radiologist trained to read MRDTI scans who were blinded to venography results. Venograms were interpreted by an independent radiologist and used as the diagnostic standard against which MRDTI was compared.

\section{Main outcome measures}

Sensitivity, specificity, and likelihood ratios for all DVT, isolated calf DVT, and proximal DVT, which included femoropopliteal and ileofemoral DVT.

\section{Main results}

The sensitivity, specificity, and likelihood ratios for DVT with MRDTI testing done by the experienced radiologist are in the table. The results for the non-radiologist reader were similar to those of the radiologist $(\kappa=0.94$, $95 \%$ CI 0.88 to 1.00$)$.

\section{Conclusion}

In patients with suspected deep venous thrombosis, magnetic resonance direct thrombus imaging was accurate and reproducible in diagnosing isolated calf and proximal deep venous thrombosis.

\section{COMMENTARY}

Ultrasonography is the diagnostic test of choice for suspected DVT. ${ }^{1}$ Examination of the femoral and popliteal veins, which are involved in $80 \%$ of symptomatic DVTs, is accurate and easy to do. ${ }^{1}$ Examination of the pelvic and calf veins is less accurate and more difficult to do. However, these veins do not need to be examined routinely because isolated iliac vein thrombosis (ie, without involvement of the common femoral vein) is uncommon (probably $<1 \%$ of DVT) and isolated calf DVT is associated with a low risk for pulmonary embolism provided that extension to the proximal veins does not subsequently occur. Because ultrasonography is unable to conclusively "rule out" DVT at presentation, a repeated ultrasonographic examination after 1 week is often required to exclude extending thrombosis. ${ }^{1}$

Fraser et al have shown that MRDTI is accurate for the diagnosis of symptomatic DVT. A normal MRDTI excluded isolated calf DVT and, although it was not specifically reported in this study, the test is also likely to exclude isolated iliac DVT. These results indicate substantial advantages over ultrasonography because they obviate the need for a repeated examination after 1 week. Another potential advantage of MRDTI is that accurate diagnosis of isolated calf DVT (which is present in about 5\% of patients with suspected DVT) would enable treatment with anticoagulant treatment. Such treatment improves short term symptoms and may reduce the long term risk for recurrent venous thromboembolism and the post-thrombotic syndrome. ${ }^{2}$ Consequently, routine diagnosis and treatment of symptomatic isolated calf DVT are expected to be preferable to only treating initially isolated calf DVT that is initially isolated but is found to have extended on a follow up ultrasonographic examination of the proximal veins. If further studies confirm that MRDTI can reliably diagnose and exclude all lower limb DVT, and if this technique becomes accessible and affordable, it will be an important advance in the diagnosis of venous thrombosis.

Clive Kearon, $\mathrm{MB}, \mathrm{PhD}$ Henderson General Hospital, McMaster Clinic Hamilton, Ontario, Canada

1 Kearon C, Julian JA, Newman TE, et al. Noninvasive diagnosis of deep venous thrombosis. McMaster Diagnostic Imaging Practice Guidelines Initiative. Ann Intern Med 1998; 128:663-77.

2 Lagerstedt CI, Olsson CG, Fagher BO, et al. Need for long-term anticoagulant treatment in symptomatic calf-vein thrombosis. Lancet 1985;2:515-8. 